\title{
Asymmetry in energy versus spin transport in certain interacting disordered systems
}

\author{
J. J. Mendoza-Arenas, ${ }^{1,2,{ }^{*}}$ M. Žnidarič ${ }^{3}$ V. K. Varma, ${ }^{4,5}$ J. Goold, ${ }^{6}$ S. R. Clark, ${ }^{7,8}$ and A. Scardicchio ${ }^{9,10}$ \\ ${ }^{1}$ Departamento de Física, Universidad de los Andes, A.A. 4976, Bogotá D. C., Colombia \\ ${ }^{2}$ Clarendon Laboratory, University of Oxford, Parks Road, Oxford OX1 3PU, United Kingdom \\ ${ }^{3}$ Physics Department, Faculty of Mathematics and Physics, University of Ljubljana, 1000 Ljubljana, Slovenia \\ ${ }^{4}$ College of Staten Island and Graduate Center, CUNY, Staten Island, New York 10314, USA \\ ${ }^{5}$ Department of Physics and Astronomy, University of Pittsburgh, Pittsburgh, Pennsylvania 15260, USA \\ ${ }^{6}$ School of Physics, Trinity College Dublin, Dublin 2, Ireland \\ ${ }^{7}$ H.H. Wills Physics Laboratory, University of Bristol, Bristol BS8 1TL, United Kingdom \\ ${ }^{8}$ Max Planck Institute for the Structure and Dynamics of Matter, CFEL, 22761 Hamburg, Germany \\ ${ }^{9}$ Abdus Salam ICTP, Strada Costiera 11, 34151 Trieste, Italy \\ ${ }^{10}$ INFN, Sezione di Trieste, Via Valerio 2, 34126 Trieste, Italy
}

(Received 9 May 2018; revised manuscript received 23 January 2019; published 22 March 2019)

\begin{abstract}
We study energy transport in disordered $X X Z$ spin- $1 / 2$ chains driven to nonequilibrium configurations by thermal reservoirs of different temperatures at the boundaries, using large-scale matrix product simulations. In particular we discuss the transition between diffusive and subdiffusive transport in sectors of zero and finite magnetization at high temperature. At large anisotropies we find that diffusive energy transport prevails over a large range of disorder strengths, which is in contrast to spin transport that is subdiffusive in the same regime for weak disorder. However, at finite magnetization both energy and spin currents decay as a function of the system size with the same exponent. We conclude that diffusion of energy is much more pervasive than that of magnetization in these disordered spin- $1 / 2$ systems, and occurs across a significant range of the interactiondisorder parameter phase space. We support the existence of this asymmetry, reminiscent of that in the clean limit, by an analytical estimation of diffusion constants for weak disorder.
\end{abstract}

DOI: 10.1103/PhysRevB.99.094435

\section{INTRODUCTION}

What determines transport of conserved quantities in generic one-dimensional disordered systems? In contrast to classical systems, where diffusion is prevalent, there is no universal answer to this question for quantum systems [1] where nonstandard hydrodynamical behaviors emerge frequently $[2,3]$. In the present paper, we address this by focusing on the well-known disordered $X X Z$ model of interacting spins, where the effect of weak disorder has been shown to result in a slow propagation of excitations [4-9]. Moreover, for stronger disorder, all forms of transport vanish, and the system transits into a many-body localized phase, where ergodicity is lost in favor of a robust integrable phase [10-17].

Slow transport can occur for both spin [4-7,9] and energy [8,18-20] (the only two conserved quantities of the model). It has also been argued that coupled particle-energy diffusion is unstable with disorder, resulting in slow dynamics [21]. Although the transport of these quantities has been suggested before to be different $[8,20]$, this has not been unambiguously demonstrated. To settle this question, we employ a configuration in which unequal baths are coupled at the ends of an archetypal interacting-disordered system to drive energy and/or spin currents. With this approach we access large system sizes $L \approx O\left(10^{2}\right)$ and thereby clearly unveil the pivotal

*jj.mendoza@uniandes.edu.co role of interactions in establishing the asymmetry between energy and spin transport.

Anomalous transport (faster or slower than diffusive) in quantum mechanical Hamiltonian systems has been associated with various properties: eigenfunction and spectral fractality [22-24], conservation laws or their approximate emergence due to frustrating dynamical constraints [25-27], and the presence of rare regions in disordered systems [15,28]. In the clean $X X Z$ spin-1/2 chain, which has an infinite set of conserved operators, the energy current operator is a conserved quantity [25]. This implies that the energy current does not decay, with its current-current correlation function persisting to a plateau in the long time limit, giving rise to a Drude peak characteristic of ballistic transport [25,29-37].

The situation for spin conduction in the clean $X X Z$ model is different. While the spin current is not a conserved quantity, a Mazur's inequality [25,38] resulting from nonlocal conservation laws of the model may be invoked to show that high-temperature transport is ballistic for anisotropy $\Delta<1$ $[26,27]$. On the other hand, evidence of superdiffusion for $\Delta=1$ and of diffusion for $\Delta>1$ has been obtained numerically $[4,39-46]$ and analytically $[47,48]$. For nonzero magnetization, however, spin transport is always ballistic due to finite overlap of the spin current with conserved quantities [25].

In the present work we find that upon the introduction of disorder, spin and energy transport maintain a relation to each other similar to that in the clean limit. Namely, if energy and magnetization flow differently (or similarly) in 
(a)

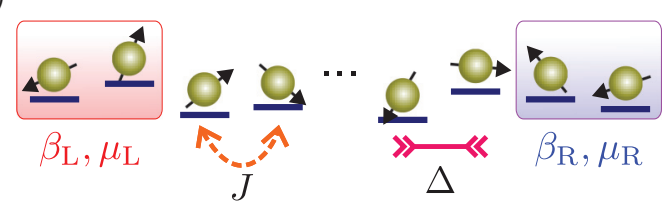

(b)

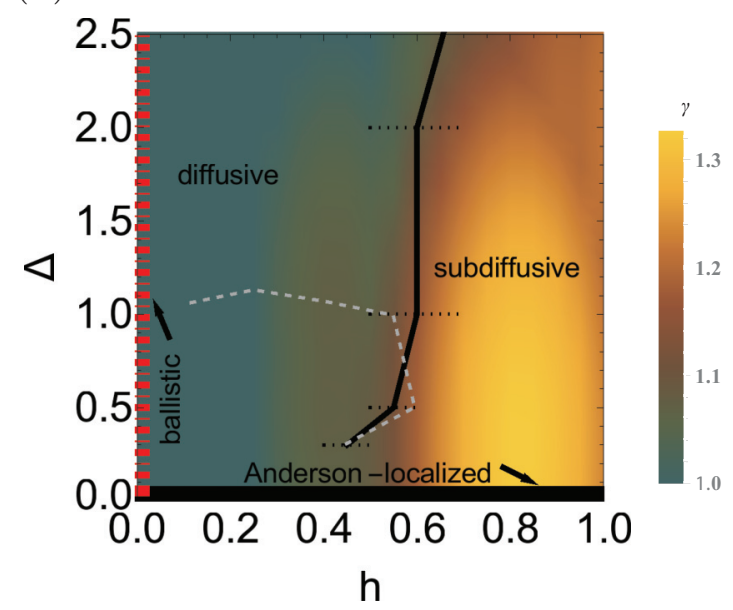

FIG. 1. (a) Scheme of a disordered $X X Z$ spin- $1 / 2$ chain driven out of equilibrium by unequal boundary reservoirs, which impose a temperature and/or chemical potential imbalance by inducing different two-site thermal states at each edge. (b) Energy transport phase diagram, indicated by the current scaling exponent $\gamma$ as a function of interaction $\Delta$ and disorder $h$. The solid black line defines the diffusive-subdiffusive boundary for zero magnetization and the dotted horizontal lines are the associated error bars. The underlying colors are Gaussian extrapolation of data, with brighter colors $(\gamma>1$, orange) indicating subdiffusion and darker colors $(\gamma=1$, green) diffusion. The gray dashed line is the diffusive-subdiffusive crossover for zero-magnetization spin transport [4].

the clean limit, then this is retained in the disordered chain. We also show that the asymmetry between both types of transport, characteristic of zero magnetization, can be drawn from an analytical estimation of diffusion constants based on an expansion of current autocorrelations into low-order moments.

\section{MODEL AND METHOD}

Here we describe the nonequilibrium setup used to study transport across a disordered quantum system, depicted in Fig. 1(a). We consider a 1D spin-1/2 lattice modeled by a $X X Z$ Hamiltonian with a disordered magnetic field along the $Z$ axis:

$$
\begin{aligned}
H & =\sum_{i=1}^{L-1}\left[J\left(s_{i}^{x} s_{i+1}^{x}+s_{i}^{y} s_{i+1}^{y}+\Delta s_{i}^{z} s_{i+1}^{z}\right)+\frac{h_{i}}{2} s_{i}^{z}+\frac{h_{i+1}}{2} s_{i+1}^{z}\right] \\
& =\sum_{i=1}^{L-1} \varepsilon_{i, i+1} .
\end{aligned}
$$

Here $L$ is the number of sites, $s_{i}^{\alpha}=\frac{1}{2} \sigma_{i}^{\alpha}(\alpha=x, y, z)$ are spin-1/2 operators for site $i$ (and $\sigma_{i}^{\alpha}$ the Pauli matrices, taking $\hbar=1), J$ is the nearest-neighbor exchange interaction, $\Delta$ is the anisotropy, $h_{i} \in[-h, h]$ is the uniformly random magnetic field at site $i$, and $h$ is the disorder strength. We set the energy scale by taking $J=1$.

To induce a temperature and/or chemical potential imbalance across the lattice, we assume that both its left $(k=\mathrm{L})$ and right $(k=\mathrm{R})$ boundaries are coupled to a reservoir characterized by an inverse temperature $\beta_{k}$ and a chemical potential $\mu_{k}$, as depicted in Fig. 1(a). The dynamics of its density matrix $\rho$ is governed by the Lindblad master equation [49]:

$$
\mathcal{L} \rho=\frac{d \rho}{d t}=-i[H, \rho]+\mathcal{L}_{\mathrm{L}}(\rho)+\mathcal{L}_{\mathrm{R}}(\rho) .
$$

$\mathcal{L}$ is the total propagator; the commutator corresponds to coherent dynamics and the terms $\mathcal{L}_{k}$ represent the incoherent action of the reservoirs on the chain. Reservoir $k=\mathrm{L}(\mathrm{R})$ is coupled to the two leftmost (rightmost) spins of the chain, in such a way that, if they were separated from the rest, a two-site nonequilibrium steady state (NESS) $\rho_{\mathrm{L}}\left(\rho_{\mathrm{R}}\right)$, corresponding to a reduced Gibbs state at an inverse temperature $\beta_{k}$, would be induced on them. These target states $\rho_{k}$ are defined in the Supplemental Material [50]; see also Refs. [37,51-53].

To characterize the nature of spin and energy transport we discuss spin and energy profiles and the NESS local currents. These are the expectation values of the local magnetization $\left\langle s_{i}^{z}\right\rangle$ and energy density $\left\langle\varepsilon_{i, i+1}\right\rangle$ for all values of $i$ and of the local current operators obtained from continuity equations [25]. The magnetization current is

$$
j_{i}^{\mathrm{S}}=\left(s_{i}^{x} s_{i+1}^{y}-s_{i}^{y} s_{i+1}^{x}\right),
$$

and the energy current is

$$
\begin{aligned}
j_{i}^{\mathrm{E}}= & {\left[\left(s_{i-1}^{y} s_{i}^{z} s_{i+1}^{x}-s_{i-1}^{x} s_{i}^{z} s_{i+1}^{y}\right)+\Delta\left(s_{i-1}^{z} s_{i}^{x} s_{i+1}^{y}-s_{i-1}^{y} s_{i}^{x} s_{i+1}^{z}\right)\right.} \\
& \left.+\Delta\left(s_{i-1}^{x} s_{i}^{y} s_{i+1}^{z}-s_{i-1}^{z} s_{i}^{y} s_{i+1}^{x}\right)+\left(h_{i} / 2\right)\left(j_{i}^{\mathrm{S}}+j_{i+1}^{\mathrm{S}}\right)\right] .
\end{aligned}
$$

These observables are determined for several disorder realizations, over which the average is performed. The NESS for each realization, defined as $\rho_{\infty}=\lim _{t \rightarrow \infty} \exp (\mathcal{L} t) \rho(0)$, is obtained with the time-dependent density matrix renormalization group [51,54-58], using a Suzuki-Trotter decomposition [59] of the Lindblad evolution operator. In the NESS both currents are homogeneous, so their disorder-averaged values are denoted by $j^{\alpha}(\alpha=\mathrm{S}, \mathrm{E})$. Details on the simulations are contained in Ref. [50].

The diffusion equation for transported quantity $A_{\alpha}$, with $A_{\mathrm{S}}=\left\langle s_{i}^{z}\right\rangle$ and $A_{\mathrm{E}}=\left\langle\varepsilon_{i, i+1}\right\rangle$, is given by

$$
j^{\alpha}=-D_{\alpha} \nabla A_{\alpha}=-D_{\alpha} \Delta A_{\alpha} / L,
$$

with $D_{\alpha}$ the corresponding diffusion constant, $\nabla A_{\alpha}$ the gradient of $A_{\alpha}$ across the chain, and $\Delta A_{\alpha}$ the difference between its boundary values. The general NESS current scaling gives $j^{\alpha} \sim \frac{1}{L^{\gamma}}$. Here $\gamma=1$ indicates normal diffusion i.e., Fick's law. When Fick's law breaks down, transport may be slower (subdiffusive, $\gamma>1$ ) or quicker (superdiffusive, $\gamma<1$ ). The scaling of the NESS current will be our primary diagonostic for inferring dynamics. We first study the case of zero magnetization, for which the main result of the diffusive-subdiffusive energy transport phase diagram is indicated in Fig. 1(b). 


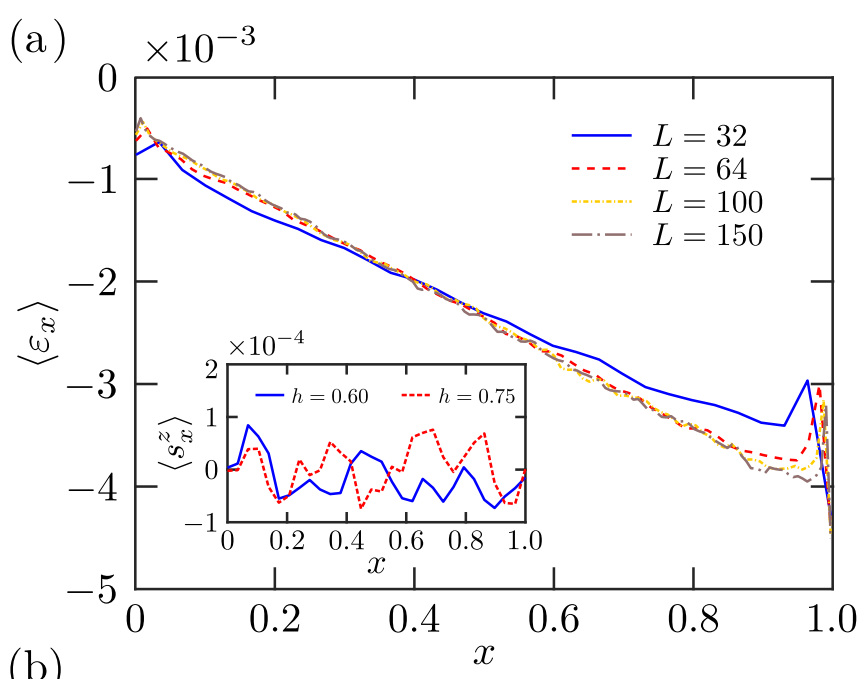

(b)

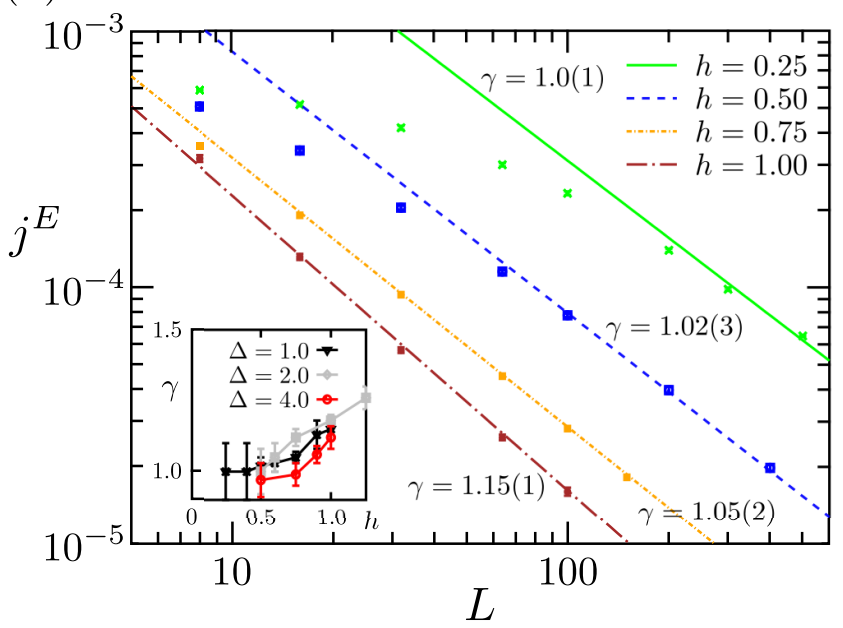

FIG. 2. NESS transport properties at zero magnetization for $\Delta=$ 1 and various disorder strengths. (a) Energy profiles for different system sizes and $h=0.60$, with scaled axis $x=(k-1) /(L-2)$ for $k=1, \ldots, L-1$. Inset: magnetization profiles for $L=32$ and different disorder strengths, with scaled axis $x=(k-1) /(L-1)$ for $k=1, \ldots, L$. (b) Scaling of energy current; the disorder strength increases from top to bottom. The symbols correspond to the results of the simulations, and the lines are fits to the scaling $j^{\mathrm{E}} \sim L^{-\gamma}$. Inset: scaling exponents $\gamma$ as a function of $h$ for different anisotropies $\Delta$.

Then we discuss nonzero magnetization. The impact of broken $\mathrm{U}(1)$ symmetry on the first setting has been considered recently [60].

\section{TRANSPORT AT ZERO MAGNETIZATION}

We first consider the case when the driving imposes a finite energy current and a negligible spin current [50]. This corresponds to an energy gradient across the lattice and almost zero total magnetization with no bias between the boundaries, as shown in Fig. 2(a).

In Fig. 2(b) we show the scaling of the NESS current for $\Delta=1$ and different disorder amplitudes. We see that diffusion persists up to a finite disorder strength, much like in the case of spin transport [4]. Then at some critical field $h_{c} \approx 0.6$ subdiffusion sets in; this value is roughly similar for the spin transport at $\Delta=1$. For larger anisotropy $\Delta>1$

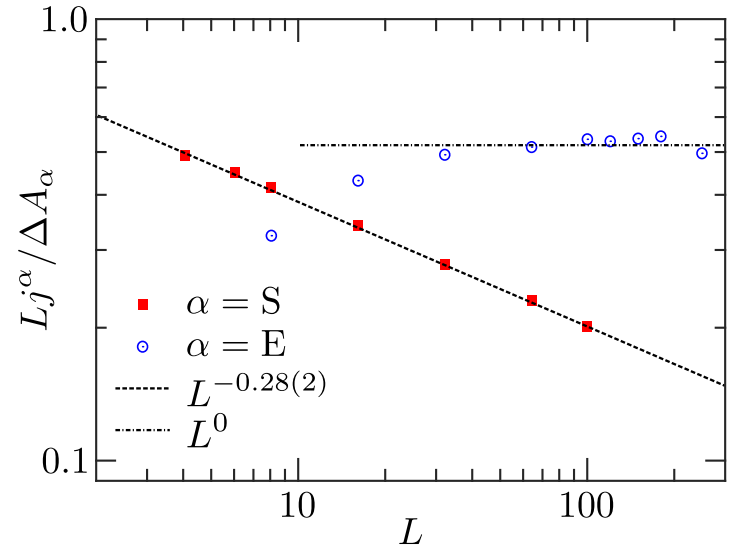

FIG. 3. Comparison of energy and spin transport in the strongly interacting case $\Delta=1.5$ and $h=0.6$, showing diffusive and subdiffusive transport, respectively. This is in qualitative agreement with the conclusions of Ref. [8] that spin and energy transport can be different in this model.

(strong interactions [50]) we also find a diffusion-subdiffusion transition at finite disorder, as shown in the inset of Fig. 2(b). However, this is entirely different to the case of spin transport, which becomes subdiffusive for much weaker (perhaps infinitesimal) disorder in this regime [4]. This effect is seen more conspicuously in Fig. 3: for the same large anisotropy $\Delta>1$, energy diffusion is clearly discernible, whereas spin transport is strongly subdiffusive.

Based on the NESS current exponents $\gamma$ extracted for several disorder and interaction strengths [50], we display a summary in the color map in Fig. 1 on a two-dimensional landscape of $h$ vs $\Delta$. We see a striking dissimilarity from that for spin transport: the phase boundary in the latter also linearly increases from the origin of this plot but buckles back towards the anisotropy line around $\Delta \approx 1$. Thus spin transport for strongly anisotropic systems immediately becomes subdiffusive upon introducing disorder, while for energy transport the diffusive-subdiffusive boundary increases all the way at least up to a strong anisotropy $\Delta=4$ [50]. We conclude that a large swath of this landscape remains diffusive for energy transport in contrast to spin conduction.

\section{MECHANISM}

This asymmetry arises from the different dynamical structure of energy and spin frequency-dependent conductivity (Fourier transform of current-current correlations) [50] in the clean limit, particularly for $\Delta \gg 1$ : there is a simple delta function at the origin for the former, but a triple-peaked structure (at $\approx 0, \pm \Delta$ ) for the latter [45]. Introducing disorder can redistribute spectral weight into the (already) dissipative components at all frequencies $\omega$ for spin but only at small $\omega$ for energy. Even using a simplified single-peak structure for spin we may account for the difference. Using a short-time expansion in disorder and interaction [50], automated with the DiracQ package [61], we find the energy and spin diffusion constants to decay as

$$
\mathcal{D}_{E} \sim \frac{J^{2} \Delta^{2}}{h}, \quad \mathcal{D}_{S} \sim \frac{J^{2}}{\sqrt{\Delta^{2}+h^{2}}}
$$



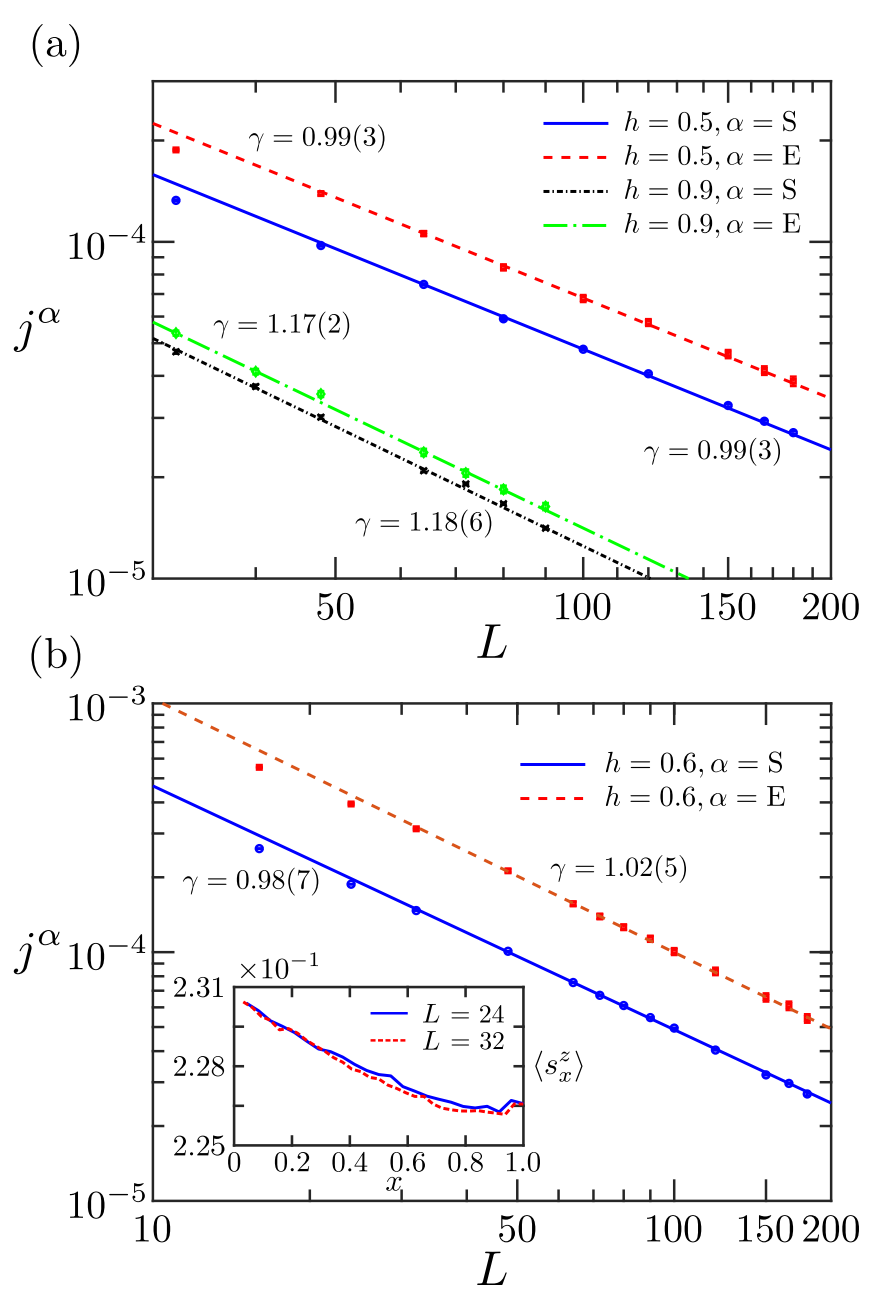

FIG. 4. NESS transport at finite magnetization. (a) Scaling of energy and spin currents for $\Delta=1$ and two different disorder strengths, indicating diffusion $(h=0.5)$ and subdiffusion $(h=0.9)$. (b) Scaling of energy and spin currents with $\Delta=1.75$ and $h=0.6$, indicating diffusion. Inset: spin profiles for different system sizes, with rescaled axis $x$.

for large $\Delta$. The nonzero diffusion constant for spin is an artifact of the short-time expansion. Nevertheless the parametric smallness of $\mathcal{D}_{S}$ compared to $\mathcal{D}_{E}$ at small $h$ is evident. This is in line with our numerics that the energy transport is "faster" than spin transport in the disordered strongly interacting case.

\section{TRANSPORT AT FINITE MAGNETIZATION}

In contrast to the zero magnetization case, in a clean system and in any finite magnetization sector both spin and energy transports are ballistic for any $\Delta$, as known from conservationlaw arguments [25] and numerical calculations [31,37,62-65]. Whether both quantities show the same transport in the presence of disorder is a natural question which we discuss in the following.

For this scenario we impose a driving that induces a finite magnetization on every site, in addition to the temperature imbalance [50]. This leads to a sizable spin current coexisting with the energy current, as shown in the main panels of Fig. 4, and a corresponding magnetization gradient across the lattice, as depicted in the inset of Fig. 4(b). The current scaling for $\Delta=1$ is shown in Fig. 4(a). As for zero magnetization at the isotropic point, both currents scale similarly as a function of disorder strength ( $h=0.5$ and $h=0.9$ are displayed), signaling equal transport dynamics (diffusion or subdiffusion for the two $h$ values, respectively) for energy and spin.

For $\Delta>1$ a similar conclusion holds, in stark contrast to zero magnetization. Namely, as illustrated for $\Delta=1.75$ and $h=0.6$ in Fig. 4(b), we have spin and energy diffusion. We note that for stronger disorder both spin and energy transport become simultaneously subdiffusive. The critical value at which this happens will be determined in a future study, but preliminary simulations show that $\gamma>1$ for $h=1$, indicating subdiffusion.

The overarching conclusion of our work is therefore that when breaking the integrability of the $X X Z$ model with weak disorder, both the asymmetry in conduction of the two conserved quantities at zero magnetization, and their similarity at finite magnetization, are maintained. Specifically, weak disorder slows the transport by a "single step" from the clean limit. This is different to the action of another integrability-breaking term, namely a weak staggered magnetic field $[37,40,51,66]$, where all transport becomes diffusive. Whether other terms such as interchain or next-nearestneighbor coupling $[32,67-69]$ also break the asymmetry is yet to be determined.

\section{EXPERIMENTAL REALIZATION}

Finally we comment on a very promising architecture for observing the discussed phenomena in the laboratory. It consists of a highly controllable and optimally measurable scheme of two unequal reservoirs of cold atoms connected through a low-dimensional channel [70]. With this setup particle currents induced by a chemical potential bias have been analyzed [71-73], even with disorder [74]. It has also been exploited to induce energy and thermoelectric transport by a temperature imbalance [75]. Furthermore, by projecting optical barriers on top of the channel, a mesoscopic onedimensional lattice was engineered, whose conduction properties were characterized in the presence of particle-particle interactions [76]. By combining and extending these techniques, it would be possible to induce particle and energy transport through disordered interacting lattices of tens of sites, enabling the predictions of our work to be verified.

\section{CONCLUSIONS}

We have studied the NESS of boundary-driven interacting and disordered spin- $1 / 2$ chains. The drives were designed to induce a temperature gradient and an associated energy current across the lattice. In the zero magnetization sector (and far from localization) we found a regime of diffusive energy transport separated from a subdiffusion regime at finite disorder strength. Moreover, for strong interactions there is a phase where energy transport is diffusive but the spin conduction is subdiffusive. However, at finite magnetization 
their dynamics are found to be the same, whether at strong or weak anisotropies. Thus weak disorder maintains the absence or presence of symmetry between spin and energy transport existing in the clean limit.

Our work paves the way for the study of more realistic models such as the disordered Hubbard lattice [77], where asymmetries between more conserved quantities (spin, charge, energy) might be discerned. We also expect that our results inspire research on their mechanism [28], and on quantum thermodynamics applications such as rectification schemes [78] and thermoelectricity [79].

\section{ACKNOWLEDGMENTS}

The authors would like to acknowledge the use of the University of Oxford Advanced Research Computing (ARC) facility in carrying out this work [80]. This research is partially funded by the European Research Council under the European Union's Seventh Framework Programme (FP7/2007-2013)/ERC Grant Agreement No. 319286 QMAC. This work was also supported by the Engineering and Physical Sciences Research Council (EPSRC) National Quantum Technology Hub in Networked Quantum Information Processing (NQIT) Grant No. EP/M013243/1. This project also received funding from the European Research Council (ERC) under the European Union's Horizon 2020 research and innovation program (Grant Agreement No. 758403). J.J.M.-A. acknowledges financial support from Vicerrectoría de Investigaciones through UniAndes-2015 project Quantum control of nonequilibrium hybrid systems-Part II. S.R.C. gratefully acknowledges support from the UK's EPSRC under Grant No. EP/P025110/1. J.G. is supported by a Science Foundation of Ireland (SFI) Royal Society University Research Fellowship. A.S. is partially supported by a Google Faculty Award. We acknowledge helpful discussions with A. Dymarsky, D. A. Huse, V. Oganesyan, and P. Prelovšek.
[1] P. W. Anderson, Phys. Rev. 109, 1492 (1958).

[2] P. M. Chaikin and T. C. Lubensky, Principles of Condensed Matter Physics (Cambridge University Press, Cambridge, UK, 1995).

[3] D. Forster, Hydrodynamic Fluctuations, Broken Symmetry, and Correlation Functions (W. A. Benjamin, Inc., New York, 1975).

[4] M. Žnidarič, A. Scardicchio, and V. K. Varma, Phys. Rev. Lett. 117, 040601 (2016).

[5] K. Agarwal, S. Gopalakrishnan, M. Knap, M. Müller, and E. Demler, Phys. Rev. Lett. 114, 160401 (2015).

[6] Y. Bar Lev, G. Cohen, and D. R. Reichman, Phys. Rev. Lett. 114, 100601 (2015).

[7] D. J. Luitz, N. Laflorencie, and F. Alet, Phys. Rev. B 93, 060201 (2016).

[8] V. K. Varma, A. Lerose, F. Pietracaprina, J. Goold, and A. Scardicchio, J. Stat. Mech. (2017) 053101.

[9] M. Žnidarič, J. J. Mendoza-Arenas, S. R. Clark, and J. Goold, Ann. Phys. (NY) 529, 1600298 (2017).

[10] R. Steinigeweg, J. Gemmer, and M. Michel, EPL 75, 406 (2006).

[11] D. Basko, I. Aleiner, and B. Altshuler, Ann. Phys. (NY) 321, 1126 (2006).

[12] V. Oganesyan and D. A. Huse, Phys. Rev. B 75, 155111 (2007).

[13] A. Pal and D. A. Huse, Phys. Rev. B 82, 174411 (2010).

[14] D. A. Abanin and Z. Papić, Ann. Phys. (NY) 529, 1700169 (2017).

[15] K. Agarwal, E. Altman, E. Demler, S. Gopalakrishnan, D. A. Huse, and M. Knap, Ann. Phys. (NY) 529, 1600326 (2017).

[16] J. Z. Imbrie, V. Ros, and A. Scardicchio, Ann. Phys. (NY) 529, 1600278 (2017).

[17] D. A. Abanin, E. Altman, I. Bloch, and M. Serbyn, arXiv:1804.11065.

[18] R. Vosk, D. A. Huse, and E. Altman, Phys. Rev. X 5, 031032 (2015).

[19] A. C. Potter, R. Vasseur, and S. A. Parameswaran, Phys. Rev. X 5, 031033 (2015).

[20] S. Gopalakrishnan, K. Agarwal, E. A. Demler, D. A. Huse, and M. Knap, Phys. Rev. B 93, 134206 (2016).

[21] P. Prelovšek and J. Herbrych, Phys. Rev. B 96, 035130 (2017).
[22] F. Piéchon, Phys. Rev. Lett. 76, 4372 (1996).

[23] R. Ketzmerick, K. Kruse, S. Kraut, and T. Geisel, Phys. Rev. Lett. 79, 1959 (1997).

[24] V. K. Varma, C. de Mulatier, and M. Žnidarič, Phys. Rev. E 96, 032130 (2017).

[25] X. Zotos, F. Naef, and P. Prelovšek, Phys. Rev. B 55, 11029 (1997).

[26] T. Prosen, Phys. Rev. Lett. 106, 217206 (2011).

[27] T. Prosen and E. Ilievski, Phys. Rev. Lett. 111, 057203 (2013).

[28] A. Nahum, J. Ruhman, and D. A. Huse, Phys. Rev. B 98, 035118 (2018).

[29] A. Klümper and K. Sakai, J. Phys. A: Math. Gen. 35, 2173 (2002).

[30] E. Orignac, R. Chitra, and R. Citro, Phys. Rev. B 67, 134426 (2003).

[31] K. Louis and C. Gros, Phys. Rev. B 67, 224410 (2003).

[32] F. Heidrich-Meisner, A. Honecker, D. C. Cabra, and W. Brenig, Phys. Rev. B 68, 134436 (2003).

[33] F. Heidrich-Meisner, A. Honecker, and W. Brenig, Eur. Phys. J.: Spec. Top. 151, 135 (2007).

[34] S. Langer, M. Heyl, I. P. McCulloch, and F. Heidrich-Meisner, Phys. Rev. B 84, 205115 (2011).

[35] C. Karrasch, R. Ilan, and J. E. Moore, Phys. Rev. B 88, 195129 (2013).

[36] C. Karrasch, D. M. Kennes, and F. Heidrich-Meisner, Phys. Rev. B 91, 115130 (2015).

[37] J. J. Mendoza-Arenas, S. R. Clark, and D. Jaksch, Phys. Rev. E 91, 042129 (2015).

[38] P. Mazur, Physica 43, 533 (1969).

[39] K. Fabricius and B. M. McCoy, Phys. Rev. B 57, 8340 (1998).

[40] G. Benenti, G. Casati, T. Prosen, D. Rossini, and M. Žnidarič, Phys. Rev. B 80, 035110 (2009).

[41] M. Žnidarič, Phys. Rev. Lett. 106, 220601 (2011).

[42] J. J. Mendoza-Arenas, T. Grujic, D. Jaksch, and S. R. Clark, Phys. Rev. B 87, 235130 (2013).

[43] J. J. Mendoza-Arenas, S. Al-Assam, S. R. Clark, and D. Jaksch, J. Stat. Mech. (2013) P07007.

[44] M. Ljubotina, M. Znidaric, and T. Prosen, Nat. Commun. 8, 16117 (2017). 
[45] R. J. Sánchez, V. K. Varma, and V. Oganesyan, Phys. Rev. B 98, 054415 (2018).

[46] R. J. Sánchez and V. K. Varma, Phys. Rev. B 96, 245117 (2017).

[47] J. De Nardis, D. Bernard, and B. Doyon, Phys. Rev. Lett. 121, 160603 (2018).

[48] E. Ilievski, J. De Nardis, M. Medenjak, and T. Prosen, Phys. Rev. Lett. 121, 230602 (2018).

[49] H.-P. Breuer and F. Petruccione, The Theory of Open Quantum Systems (Oxford University Press, Oxford, 2002).

[50] See Supplemental Material at http://link.aps.org/supplemental/ 10.1103/PhysRevB.99.094435 for specifics of the implementation and simulation of the driving scheme, supporting data of current scaling and energy profiles, and details on the short-time expansion.

[51] T. Prosen and M. Žnidarič, J. Stat. Mech. (2009) P02035.

[52] M. Žnidarič, T. Prosen, G. Benenti, G. Casati, and D. Rossini, Phys. Rev. E 81, 051135 (2010).

[53] M. Žnidarič, J. Stat. Mech. (2011) P12008.

[54] M. Zwolak and G. Vidal, Phys. Rev. Lett. 93, 207205 (2004).

[55] F. Verstraete, J. J. Garcia-Ripoll, and J. I. Cirac, Phys. Rev. Lett. 93, 207204 (2004).

[56] U. Schollwöck, Ann. Phys. (NY) 326, 96 (2011).

[57] S. Al-Assam, S. R. Clark, and D. Jaksch, J. Stat. Mech. (2017) 093102.

[58] S. Al-Assam, S. R. Clark, D. Jaksch, and T. Development Team, Tensor Network Theory Library, Beta Version 1.2.0, 2016, http://www.tensornetworktheory.org.

[59] M. Suzuki, Phys. Lett. A 146, 319 (1990).

[60] M. Schulz, S. R. Taylor, C. A. Hooley, and A. Scardicchio, Phys. Rev. B 98, 180201 (2018).

[61] J. Wright and B. S. Shastry, DiracQ: A Quantum Many-Body Physics Package, arXiv:1301.4494.

[62] F. Heidrich-Meisner, A. Honecker, and W. Brenig, Phys. Rev. B 71, 184415 (2005).
[63] K. Sakai and A. Klümper, J. Phys. Soc. Jpn. Suppl. 74, 196 (2005).

[64] S. Furukawa, D. Ikeda, and K. Sakai, J. Phys. Soc. Jpn. 74, 3241 (2005).

[65] S. Ajisaka, F. Barra, C. Mejí-Monasterio, and T. Prosen, Phys. Rev. B 86, 125111 (2012).

[66] R. Steinigeweg, J. Gemmer, and W. Brenig, Phys. Rev. B 91, 104404 (2015).

[67] P. Jung, R. W. Helmes, and A. Rosch, Phys. Rev. Lett. 96, 067202 (2006).

[68] M. Žnidarič, Phys. Rev. B 88, 205135 (2013).

[69] C. Karrasch, J. E. Moore, and F. Heidrich-Meisner, Phys. Rev. B 89, 075139 (2014).

[70] S. Krinner, T. Esslinger, and J.-P. Brantut, J. Phys.: Condens. Matter 29, 343003 (2017).

[71] J.-P. Brantut, J. Meineke, D. Stadler, S. Krinner, and T. Esslinger, Science 337, 1069 (2012).

[72] D. Stadler, S. Krinner, J. Meineke, J.-P. Brantut, and T. Esslinger, Nature (London) 491, 736 (2012).

[73] S. Krinner, D. Stadler, D. Husmann, J.-P. Brantut, and T. Esslinger, Nature (London) 517, 64 (2015).

[74] S. Krinner, D. Stadler, J. Meineke, J.-P. Brantut, and T. Esslinger, Phys. Rev. Lett. 110, 100601 (2013).

[75] J.-P. Brantut, C. Grenier, J. Meineke, D. Stadler, S. Krinner, C. Kollath, T. Esslinger, and A. Georges, Science 342, 713 (2013).

[76] M. Lebrat, P. Grišins, D. Husmann, S. Häusler, L. Corman, T. Giamarchi, J.-P. Brantut, and T. Esslinger, Phys. Rev. X 8, 011053 (2018).

[77] M. Kozarzewski, P. Prelovšek, and M. Mierzejewski, Phys. Rev. Lett. 120, 246602 (2018).

[78] M. Perarnau-Llobet, H. Wilming, A. Riera, R. Gallego, and J. Eisert, Phys. Rev. Lett. 120, 120602 (2018).

[79] G. Benenti, G. Casati, K. Saito, and R. S. Whitney, Phys. Rep. 694, 1 (2017).

[80] http://dx.doi.org/10.5281/zenodo.22558. 